\title{
WOLFRAM SYNDROME IN A FAMILY WITH VARIABLE EXPRESSION
}

\author{
Abdurrahman Kadayifci ${ }^{1}$, Yalcin Kepekci ${ }^{1}$, Yavuz Coskun ${ }^{2}$ and Ying Huang ${ }^{3}$
}

Gaziantep University, Faculty of Medicine, Turkey: Department of Internal Medicine ${ }^{1}$, Department of Pediatrics ${ }^{2}$; New York Medical College, Hawthorne, New York, USA: The Institute for the Genetic Analysis of Common Diseases ${ }^{3}$

Summary: Wolfram syndrome is a rare neurodegenerative disorder with autosomal recessive inheritance. The main characteristic features of this disorder are diabetes mellitus and optic atrophy. However, diabetes insipidus, sensorineural deafness, renal tract and neurologic abnormalities are seen in majority of patients. In this study, we describe a family in which two members had the main features of the syndrome while a third sibling had only sensorineural deafness. DNA analysis revealed that the fully affected siblings were homozygote for a pointmutation on chromosome $4 \mathrm{p}$ whereas the third sibling with deafness was a heterozygote carrier for the same mutation. The characteristics of disease and phenotypic variations that possibly related to heterozygote carrier state were discussed.

Key words: Wolfram syndrome; DIDMOAD syndrome; Heterozygote carriers; Mutation

\section{Introduction}

The Wolfram Syndrome that is also known as DIDMOAD syndrome is characterized with the presence of diabetes insipidus, diabetes mellitus, optic atrophy and deafness resulting from a degenerative process involving pancreatic $\beta$ cells, supraoptic and paraventricular nuclei, the optic nerve, and cranial nerve VIII. Dilatations in urogenital system and variable neurological and psychiatric abnormalities may be associated with the disease. The inheritance of the syndrome is autosomal recessive and typical findings are only expected in homozygotes for disorder $(6,9,12)$. Recent studies showed the gene of Wolfram syndrome is linked to the markers on the short arm of chromosome $4(3,7)$.

This paper describes a family with six siblings of whom the first one has full expression of Wolfram syndrome; diabetes insipidus, diabetes mellitus, optic atrophy, sensorineural deafness and urinary dilatation, the second one presents with diabetes insipidus (incomplete), diabetes mellitus, visual disturbance and deafness, the third one with only sensorineural hearing loss, and the fourth sibling is prediabetic and has early coronary heart disease whereas the other two siblings are healthy. The parents are first cousins and have no health problem. The incomplete findings of Wolfram syndrome in some individuals of this family were highly interesting. To contribute the clarifying the genetic and phenotypic variations of this rare syndrome, we investigated this family with a detailed clinical examination and DNA analysis.

\section{Patients and Methods}

\section{Cases}

Case One: An eighteen-year-old diabetic male patient was admitted with complaints of urinary retention and incontinence starting three months prior to admission. The patient had been given insulin therapy for diabetes mellitus for five years. From the history, the patient complained of polydipsia exceeding 8-10 liters a day. Physical examination was normal exception dehydration, glove and sock like hypoestesia in the distal part of the extremities and hypoactivity in the deep tendon reflexes. Myopia, optic atrophy, posterior polar cataract and strabismus were detected in the ophthalmologic examination. Case Two: Twenty-nine year old male patient had Type 1 diabetes mellitus for ten years and were using insulin with poor compliance to treatment. He also had hearing loss and visual disturbances. Physical examination revealed 2/6 diastolic murmur, absence of deep tendon reflexes, glove and sock like hypoestesia in the extremities. Eye findings were myopia and marked pallor in the optic discs. Case Three: This sibling is a thirty-two year old female. She had only complete deafness. From her history, it was learned that she had congenital deafness. There was no history of congenital rubella or any other neonatal infections, anoxia during birth, trauma to skull, and central nerve system infection. Because she had congenital deafness and was given no special training, she did not develop speech normally. Therefore, she had mutism. In clinical examination, the Weber and Rinne tests revealed bilateral sensorineural hearing loss. Case 
Four: Thirty-seven year old man had chest pain suggesting angina pectoris. He had not a major risk factor of coranary heart disease in history and physical examination. The other two siblings are a 22 year-old woman and 28 year-old man. The fifth sibling was no abnormality in history and physical examination. The sixth sibling has not been examined since he was living another city but it was learned from other family member that he had not a known health problem.

The history and examination of affected cases and similar health problems among the siblings suggested a genetic disorder in this family that consistent with Wolfram syndrome. So, all siblings and parents underwent a detailed laboratory examination for diabetes mellitus, diabetes insiputus, hearing loss and renal tract abnormalities.

It has been learned from the family history that grandfather of these patients had committed suicide. No severe mental illness was detected on the psychiatric examination of cases but a tendency to depression on the first case and some changes in character on the second case were observed.

\section{DNA analysis}

For DNA analysis, blood samples of a living grandmother, parents and all siblings (except only sixth sibling) were collected in tubes with EDTA. Genomic DNA was isolated from peripheral blood using standard procedures. Mutations present in the WS patients or their parents (obligate carriers) were identified through direct sequencing of the WS locus from proband or parental genomic DNA. Each exon of the WS gene and its splicing sites was amplified from genomic DNA by the polymerase chain reaction (PCR) using a GeneAmp PCR System 9600 (PE Applied Biosystems, Foster City, CA). The PCR products were directly sequenced by the Big Dye Primer Cycle Sequencing Reactions using the ABI 877 Integrated Thermal Cycler and the ABI 377 Automated Sequencer (Perker-Elmer Applied Biosystems, CA). A variation from the WS DNA sequence in GenBank was determined to be a true mutation if there was a corresponding change in the amino acid sequence. Once a specific mutation was identified in the parental DNA, the appropriate region of the WS gene was then amplified and sequenced for the other relatives. Those relatives showing the WS mutation found in the parents were considered carriers.

\section{Results}

The following results were found in the laboratory tests of cases.

Case One: Laboratory findings revealed that blood glucose level $374 \mathrm{mg} / \mathrm{dl}$, hemoglobin A1c $13 \%$, urinary gravity 1005 , urinary osmolality $155 \mathrm{mOsm} / \mathrm{kg}$, and plasma osmolality $306 \mathrm{mOsm} / \mathrm{kg}$. Urinary osmolality did not show any marked increase during a three hours water deprivation test while the plasma osmolality went on to increase.
Urinary osmolality started to increase after a $10 \mu \mathrm{g}$ desmopressin by nasal spray (Table 1). Abdominal ultrasonography and intravenous pyelography showed increased echogenity in the parenchyma of both kidneys and dilatation in pelvicaliciel systems and ureters as well as increased residual volume in the bladder. Cranial computed tomography was normal. A mild sensorineural hearing loss (30 $\mathrm{dB}$ on the right and $22 \mathrm{~dB}$ on the left) was found in audiometric examination.

Tab. 1: The results of water deprivation test in case 1 and 2 showing diabetes insipidus.

\begin{tabular}{|c|c|c|c|c|}
\hline & \multicolumn{2}{|c|}{ Case 1 } & \multicolumn{2}{c|}{ Case 2 } \\
\hline Hours & Posm & Uosm & Posm & Uosm \\
\hline $\mathbf{0}$ & 306 & 147 & 291 & 115 \\
\hline $\mathbf{1}$ & 310 & 154 & 296 & 149 \\
\hline $\mathbf{2}$ & 315 & 166 & 302 & 186 \\
\hline $\mathbf{3}$ & 327 & 142 & 310 & 281 \\
\hline $\mathbf{4}^{*}$ & 312 & 423 & 304 & 675 \\
\hline
\end{tabular}

*: The samples obtained after 1 hour of $10 \mu \mathrm{g}$ desmopressin nasal spray

The blood sugar of patient was regulated with appropriate insulin doses. The clinical findings of diabetes insipidus improved dramatically within two weeks. At first, continuous urinary cathaterisations were necessary for voiding disturbances. The patient is now having intermittent urinary cathaterisations three or five times a day without urinary retention and incontinence.

Case Two: Laboratory investigations were normal except a blood glucose level of $304 \mathrm{mg} / \mathrm{dl}$ and slight decrease in urinary gravity (1006) and urinary osmolality $(166 \mathrm{mOsm} / \mathrm{kg})$ with an increase in plasma osmolality $(305 \mathrm{mOsm} / \mathrm{kg})$. Water deprivation test of this patient showed a slight increase in urinary osmolality suggesting an incomplete diabetes insipudus (Table 1). The use of chlorpropamide improved the symptoms of patients and urinary osmolality as well. Echocardiography showed moderate aortic insufficiency. Abdominal ultrasonography revealed no abnormality. Total hearing loss was found on audiometric investigation.

Case Three: Laboratory examinations including biochemical, urine analysis and abdominal ultrasonography were normal. The audiometric investigation confirmed Weber and Rinne tests and showed bilateral severe sensorineural hearing loss.

Case Four: He had impaired glucose tolerance test with a blood glucose level of $171 \mathrm{mg} / \mathrm{dl}$ at $2 \mathrm{~h}$ of test and with a $214 \mathrm{mg} / \mathrm{dl}$ value at $1 \mathrm{~h}$. Coronary angiography had revealed an early coronary heart disease with moderate occlusion on the left coronary artery.

All of the laboratory investigations related to Wolfram syndrome revealed no abnormality in fifth siblings and parents. The clinical parameters of the all cases were summarized in the table 2 . 
Tab. 2: The clinical parameters of the siblings.

\begin{tabular}{|l|c|l|}
\hline & Age & Clinical Findings \\
\hline I & 18 & $\begin{array}{l}\text { DM, DI, OA, hearing loss, urological manifestati- } \\
\text { ons, neurological manifestations, other eye fin- } \\
\text { dings. }\end{array}$ \\
\hline II & 29 & $\begin{array}{l}\text { DM, DI (incomplete), aortic insufficiency, deaf- } \\
\text { ness, abnormal eye findings, neurological mani- } \\
\text { festations. }\end{array}$ \\
\hline III & 32 & Deafness \\
\hline IV & 36 & Prediabetic, early coronary heart disease \\
\hline
\end{tabular}

DM: Diabetes mellitus, DI: Diabetes insipidus, OA: Optic atrophy

The DNA analysis revealed a $1885 \mathrm{C}$ to $\mathrm{T}$ transversion of chromosome $4 \mathrm{p}$ in this family. This mutation leads to a change in codon 629 , so that amino acid becomes tryptophan instead of arginine. The first and second siblings were homozygous for this mutation whereas the third sibling with hearing loss was heterozygous. The parents and grandmother were also heterozygous for the same mutation. The fourth and fifth siblings were homozygous for the wild-type allele.

\section{Discussion}

Wolfram syndrome is a genetic disorder inherited in an autosomal recessive mode and it occurs in siblings of unaffected parents, which both are heterozygote carriers for the same autosomal recessive gene $(3,7)$. Autosomal recessive diseases are clinically evident only in the homozygous for the mutation found. This means both alleles at a particular genetic locus are mutant in these conditions. On the other hand the heterozygous carriers in autosomal recessive inheritance are unaffected showing that mutations in the gene cosegragate with the phenotype. Predisposition of Wolfram syndrome heterozygous to psychiatric illness as well as homozygous have been notified in some studies ( 10 , 11).The Wolfram family in this study is very interesting from this aspect. The clinical presentation of the first and second cases were typical for Wolfram syndrome but the incomplete findings in third and fourth cases were considerable interest because they do raise questions about manifestations of the Wolfram syndrome mutant allele in heterozygotes. The DNA analysis of affected and unaffected individuals clearly showed that this family had a pointmutation on chromosome $4 \mathrm{p}$ confirming Wolfram syndrome. The first two cases were homozygous for this mutation in consisted with clinical presentations. However, the third case manifested with congenital sensorineural deafness was heterozygous for the same mutation. Sensorineural hearing loss classically is not congenital in Wolfram syndrome but devolops later without mutism. So, the characteristic of sensorineural hearing loss is not accordance with classic Wolfram syndrome in this case. On the other hand, the history and detailed neurological investigations of this patient detected no cause for hearing loss. Therefore, the association of hearing loss with Wolfram is obscure in this patient. The DNA analysis detected no mutation in fourth case. Therefore, impaired OGTT test and early coronary heart disease in this patient does not seem related to Wolfram syndrome.

It is not possible to explain exactly how some manifestations of an autosomal recessive disease occur in a heterozygote carrier state. It is a probability that there may be a more complex genetic alteration rather than classical mendelian inheritance in at least some of Wolfram patients. However, this hypothesis requires more complex and further analysis of these patients. Variable manifestations among patients, even between siblings suggested that mitochondrial dysfunctions could be involved in the disease. A loss of mitochondrial function was showed in two sporadic cases $(2,8)$. Moreover, Barrientas et al showed the presence of a high proportion of deletion in mtDNA of a Wolfram patient (1). However this hypothesis has not been supported by a more recent study (4). Inove et al found mutations in a novel gene encoding a putative transmembrane protein in Wolfram patients and this appears to function in survival of islet beta-cells and neurons (5). This is an important finding to explain the different manifestations in disease phenotype but still insufficient to explain variable manifestations among siblings and in heterozygous.

Optic atrophy is a characteristic finding of Wolfram syndrome. However aside optic atrophy, we observed unusual ophthalmic findings for Wolfram patients such as posterior polar cataract and strabismus in case one, and moderate aortic insufficiency in case two. Whether these findings are related to Wolfram or simply a coincidence is unclear at present. It has been emphasized previous studies that both homozygous and also heterozygous carriers of the gene for the Wolfram is tend to significant mental disorders $(4,5)$. A depressed mood in case one and some changes in character on the second case is consistent with these reports. The committed suicide of grandfather in our family may also be related to a possible heterozygous carrier for the mutant gene of Wolfram.

Although the association is not clear, this study notices that some members of Wolfram families other than homozygous siblings may have some findings of this syndrome. Moreover, some unusual eye findings and cardiac abnormalities may be related to Wolfram syndrome. However, further studies are needed to clarify the cause of these phenotypic variations of this rare syndrome.

\section{References}

1. Barrientos A, Casademont J, Saiz A et al. Autosomal recessive Wolfram syndrome associated with an 8.5-kb mtDNA single deletion. Am J Hum Genet 1996;58:963-70.

2. Bundey S, Poulton K, Whitwell $\mathrm{H}$ et al. Mitochondrial abnormalities in the DIDMOAD syndrome. J Inherit Metab Dis 1992;15:315-9.

3. Collier DA, Barrett TG, Curtis D et al. Linkage of Wolfram syndrome to chromosome 4p16.1 and evidence for heterogeneity. Am J Hum Genet 1996;59:855-63. 
4. Hofman S, Bezold B, Jaksch M et al: Wolfram (DIDMOAD) syndrome and Leber hereditary optic neuropathy (LHON) are associated with distinct mitochondrial DNA haplotypes.Genomics 1997;1:8-18

5. Inoue $\mathrm{H}$, Tanizawa $\mathrm{Y}$, Wasson $\mathrm{J}$ et al. A gene encoding a transmenbrane protein is mutated in patients with diabetes mellitus and optic atrophy (Wolfram syndrome). Nat Genet 1998;20:143-8.

6. Perheentupa J. The neurohypophysis and water regulation. In: Brook GD, ed. Clinical Pediatric Endocrinology. London, Blackwell Scientific Publication, $2^{\text {nd }}$ Ed, 1989:296

7. Polymeropoulos MH, Swift RG, Swift M. Linkage of the gene for Wolfram syndrome to markers on the short arm of chromosome 4. Nat Genet 1994;8:95-7.

8. Rötig A, Cormier V, Chatelain P et al. Deletion of mitochondrial DNA in a case of early-onset diabetes mellitus, optic atrophy and deafness (Wolfram syndrome, MIM 222300). J Clin Invest 1993;91:1095-8.

9. Swift RG, Perkins DO, Chase CL et al. Psychiatric disorders in 36 families with Wolfram syndrome. Am J Psychiatry 1991;148:775-9.

10. Swift RG, Polymeropoulos MH, Torres R et al: Predisposition of Wolfram syndrome heterozygotes to psychiatric illness. Mol Psychiatry 1998;3:86-91.

11. Swift RG, Sadler DB, Swift M. Psychiatric findings in Wolfram syndrome homozygotes. Lancet 1990;336:667-9.

12. Tarsy D, Freeman R. The Nervous System And Diabetes. Kahn CR, Weir GC eds. Joslin's Diabetes Mellitus. Pennsylvania, Lea\&Febiger-Waverly Co, $13^{\text {nd }} \mathrm{Ed}$, 1994:808.

Submitted March 2001.

Accepted November 2001.

Dr. Abdurrahman Kadayifci,

Ömer Asim Aksoy cad. Levent apt., 37/8, Sarigulluk, Gaziantep, Turkey.

E-mail: kadayifci@gantep.edu.tr 